\title{
Enhancing healthcare professional peer support and resilience with reflective narrative panel: Innovative workshop for impacting medical setting culture
}

\author{
Emerson ND ${ }^{1}$ and Bursch B $^{2 *}$ \\ ${ }^{1}$ Psychology Intern, Department of Psychiatry \& Biobehavioral Sciences, David Geffen School of Medicine at UCLA, Los Angeles, CA, USA \\ ${ }^{2}$ Professor of Clinical Psychiatry \& Biobehavioral Sciences, Professor of Clinical Pediatrics, Clinical Director, Pediatric Psychiatry Consultation Liaison Service, \\ David Geffen School of Medicine at UCLA, Los Angeles, CA, USA
}

\begin{abstract}
Healthcare professionals are exposed to difficult situations that increase risk for burnout and secondary trauma. Despite the known benefits of peer support and reflection in promoting the resilience of workers, many employees do not self-disclose to peers due to fear of stigmatization. Exposure to stories of common challenges faced by health professionals has been shown to increase external help-seeking behavior but does not address local culture. This paper describes an innovative reflective narrative workshop format designed to address local culture by including known work group leaders in a panel discussion of their personal work-related challenges. Specific goals of the workshop are to decrease stigma related to discussion of challenging personal work-related situations, normalize discussions about the day-to-day work-related struggles, and share pearls of wisdom related to effective coping in health settings.
\end{abstract}

Healthcare professionals are exposed to difficult situations on a daily basis. Caring for medically and/or mentally ill patients often takes a toll on employees' emotional wellbeing and sense of efficacy given the natural instability of patients' health status and the challenging nature of healthcare work routines [1].

While training and education of healthcare professionals has grown to encompass the importance of self-care practices, it remains an unspoken assumption that those involved in patient care must be psychologically robust. As such, rather inadvertently, there often exists an underlying stigmatization towards showing emotional fragility and difficulty coping in the workplace.

Although there is widespread interest and progress being made related to the development of peer support programs and resilience skills training to combat health professional burnout and trauma exposure, there remains a need for interventions designed to impact local medical setting culture in order to overcome the peer support seeking obstacle of stigmatization.

\section{Burnout, Second Victim Phenomenon \& Resilience}

Both burnout and second victimhood have been used to characterize the effects of chronic exposure to acute occupational stress. Defined by exhaustion, depersonalization, and a low sense of personal accomplishment, burnout may be particularly prevalent in workers who must cope with recurrent traumatic clinical scenarios and environmental stressors of working in challenging health settings (long hours, medical hierarchy, complex cases with unclear solutions) [2,3]. In parallel, second victimhood, also known as vicarious traumatization, has been used to describe the emotional response of workers following an unexpected clinical adverse event such as patient death or injury [4]. Burnout and second victimhood are thought to be interrelated concepts, one may beget the other and vice versa. Both concepts are important to consider in health settings given that novice and experienced workers alike are more likely to commit occupational errors when burned out $[5,6]$. In addition to the deleterious consequences on quality of care, decreased patient satisfaction and increased job turnover are also observed among burned out health professionals [1].

Since exposure to difficult scenarios cannot be altogether avoided, bolstering workers' resilience is one important approach for maintaining the health of employees and optimizing occupational longevity. Resilience is defined as "the ability of an individual to bounce back from adversity, persevere through difficult times, and return to a state of internal equilibrium or a state of healthy being" [7]. Researchers who have studied the development and maintenance of professional resilience have identified the importance of interpersonal support for the sustenance of work in emotionally-laden fields. In particular, there appears to be a strong, negative relationship between social support and the burnout component of emotional exhaustion $[8,9]$. The ability to confide in and be supported by others appears to be protective against the feelings of inadequacy, guilt, and helplessness that result from interpersonally-heavy work.

Seeking help from peers within one's organization may be particularly helpful. As described by Scott and colleagues [4], second victims, if capable of asking for help following an adverse event, tend to desire and seek out support from colleagues, rather than from family

${ }^{\star}$ Correspondence to: Bursch $\mathrm{B}, \mathrm{PhD}$, Psychiatry \& Biobehavioral Sciences, UCLA Semel 48-241, Los Angeles, CA 90024-1759, USA, Tel: 310-206-4985; E-mail: bbursch@mednet.ucla.edu

Key words: burnout, narrative, peer, reflective, resilience, support

Received: May 16 2018; Accepted: May 29, 2018; Published: May 31, 2018 

culture

and friends, due to peers' ability to empathize and to restore the second victims' sense of self-efficacy by validating their clinical proficiency [4]. Moreover, resilience, like burnout, may have a contagious effect in the workplace. Supervisors and peers alike can spread a culture of well-being, self-care, and positivity by modeling personal resilience themselves [10]. Finally, receiving support from within one's organization may also mitigate against the risk of burnout and second victimhood by increasing workers' organizational identification and the associated feeling of collective efficiency [11].

\section{Reflective narrative}

Reflective narrative is a description of an experience that is presented in the form of a story. The story normally integrates the storyteller's emotions with their perceptions of salient events. The story can be verbal or written. Reflective narrative allows one to derive value and meaning from recent events. Narrative medicine is an approach to clinical care, research and education that utilizes people's reflective narratives to promote healing. Compared to a traditional biomedical approach that is focused on symptom management, narrative medicine aims to validate the experience of the patient and to encourage clinician creativity and self-reflection. Both patients and clinicians have used reflective narrative as a tool for processing complex and/or emotionally challenging situations.

When facing a life-threatening or chronic illness or disability, most patients attempt to integrate and make sense of their new reality within their life experiences to date. Reflective narrative can be a helpful tool to effectively address this psychological need. Preliminary research reveals that reflective narrative approaches that include emotional disclosure are correlated with better health outcomes, decreased pain and other somatic symptoms, increased well-being related to illness, and increased illness-related confidence, action and cooperation [12,13].

When clinicians have used reflective narrative, often within clinical training programs, the goals are typically to stimulate the development of reasoning, professionalism, insight, empathy and critical thinking by promoting the translation of thoughts and emotions into words. Additionally, the summarization and integration of thoughts and feelings required to create an intelligible narrative can help alleviate the traumatic nature of otherwise traumatizing events. Research reveals that the use of reflection among medical residents and fellows improved learning of complex subjects, deepened professional values, and improved attitudes and comfort when exploring difficult material [14].

Less studied, reflective narrative may also prove beneficial to the listener. As described earlier, despite the apparent benefit derived from workplace peer support, employees often avoid seeking help from colleagues due to fear that revelation of their own difficulties may label them as weak or incompetent [15]. Personal stories of clinicians experiencing and coping with and/or overcoming adversity have been used to increase trainees' likelihood of seeking professional assistance when needed [16]. Such stories, however, do not necessarily impact local culture related to asking for help from supervisors or colleagues.

Having leaders from one's own work group or department share stories of personal struggles using a narrative approach has the potential of impacting local culture and increasing social support seeking. The purpose of this paper is to describe an innovative educational workshop designed to impact local culture with the use of live reflective narrative storytelling by known leaders. The objectives of the intervention are to decrease stigma related to discussion of challenging personal situations, normalize discussions about the day-to-day struggles, and share pearls of wisdom related to effective coping in health settings.

\section{Innovative use of reflective narrative to impact medical setting culture}

The reflective narrative educational workshop is comprised of a panel of health professionals along with a facilitator. For a one-hour session, 4-5 storytellers are recommended. It may be helpful for the workshop planner/facilitator to solicit suggestions about desired presenters from future audience members. Generally, storytellers should be professionals who are well-respected and in leadership positions. Efforts should be made to avoid inviting younger and newer team members to share as they may feel more insecure in their positions. Consideration should be given to ensure that the panel of storytellers are as diverse as the future audience in terms of gender, professional discipline, story topics and/or other attributes identified as important. No one should be pressured to participate.

Instructions for the guest panelists are in Table 1. Panelists are instructed to prepare a brief narrative that focuses on their own emotional experience. This caveat is required to prevent participants from sharing the story from the patient or health system's perspective, which diminishes the power of the narrative reflection both in terms of its benefits to the narrator and to the audience. Panelists should be advised to focus on their emotional challenges and reactions rather than clinical or theoretical content of a scenario. Gruesome clinical details should be omitted as they may traumatize participants and take attention from the theme of meaning making and interpersonal

Table 1. Instructions to Guest Panelists

$$
\text { Instructions to Guest Panelists }
$$

Please think about some of your personally most challenging moments within your job. Events that work the best are those that others might relate to and that are difficult to discuss due to shame, embarrassment, guilt or other difficult emotions.

The goal is to share your story in the form of a very short, heartfelt, vulnerable and open description (4-5 minutes max) of one of your worst moments related to your job (without traumatizing anyone with unneeded details) and a few minutes (2-3 minutes max) on what did and did not help you cope (both external forces, such as colleagues or institutional response and your internal responses, such as positive self-talk or other helpful/ unhelpful coping or behaviors). Feel free to add any pearls of wisdom you gained from the experience, if applicable. Situations that did not resolve well are sometimes even more valuable as those that turned out okay.

Examples of Potential Topics.

- Death of a patient

- Disagreeing with colleagues or supervisor

- Feeling completely professionally inadequate

- Lawsuit or complaint filed

- Living up to high expectations

- Making an error

- Being dismissed from a project or patient

- Moral distress related to a patient or other situation

- One's own differences

- One's own medical challenge

- One's own mental health challenge

Hopefully there will be an array of topics so that the audience gets a taste of different types of struggles. Once you have tentatively picked a topic, please email me so that I can change the order of the presenters as needed based on the topics selected. Practice what you will say to ensure that it is contained to the very tight timeline that we will have. I am happy to discuss your topic with you, listen to your presentation or wait until the big day with only knowing your general topic. Up to you.

Again, please role model being open and vulnerable. You were chosen because you are viewed as highly successful with excellent coping skills. It is hard for others to imagine you struggling as they do at times. We want others to feel better (not alone) about times they too have felt similar difficult emotions. Please do not provide a didactic lecture about coping/burnout or present as an expert. The idea is to tell a narrative/story that is personal to you and briefly reflect on the event. 

culture

growth. Lastly, panelists should be encouraged to consider the audience as the most meaningful aspects of the content may be different for nonclinicians, faculty, and trainees.

The panel facilitator may wish to meet with each participant in order to help organize their narrative, highlight themes, and to ensure the story is framed as the clinician's reflective narrative. This step is not obligatory but may prove useful to the panelists given the highly emotional material they are presenting. It is easy for health professionals to slip into a more didactic role or into a clinical case presentation mode.

\section{Workshop format}

The workshop starts with a brief introduction by the panel facilitator, showcasing the theme and purpose of the panel. For instance, the leader may begin with the following introduction:

"Thank you for coming to our workshop today, designed to enhance your resilience in the workplace in order to reduce your risk of burnout and/or work-related trauma symptoms. Each panelist will share a story that reflects a particularly challenging event they experienced at work. The purpose of sharing these stories is to normalize some of the most common challenges encountered in our day-to-day work with the hopes that we will destigmatize self-disclosure of difficulty and improve supportseeking from our peers. Our hope is that, after hearing these stories, you will see that you are not alone in your struggles and that you will feel more open about asking for support from your colleagues when you are facing your own difficulties."

It is suggested that the panel facilitator request that questions and comments be left for the very end of the workshop in order to allow each audience member to personally reflect on the stories they hear.

The panel facilitator will then transition to the first storyteller, subtlety alerting them when they have 1-2 minutes left. In some settings, the panel facilitator may choose to summarize one pertinent piece of information after each story as a means of helping panelists and audience members organize their emotional reactions to the narration. This comment, if inserted, should be brief and focus on the emotional connotation of the story. Such a comment may also help naturally transition from one speaker to the next. The panel facilitator thanks each storyteller before moving on to the next one.

Once each story has been told, the panel facilitator has several options. The first option is to make any summary statements that will help the audience digest what they have heard. A second option is to ask the guest panelists how they felt when asked to present on the panel. This typically elicits statements regarding anticipatory anxiety, that further reveals the humanness of the storytellers. The third option is to open up the workshop to the audience members for their comments and questions. It is typically possible to complete all three tasks if the facilitator is succinct and able to encourage others to also be succinct.

\section{Examples}

In order to illustrate the breadth of settings and topics that can be addressed, following are two recent examples of the use of this approach in a medical setting (Table 2).

\section{Discussion}

The sharing of personal work-related struggles by respected department leaders may help to destigmatize social support-seeking behaviors among health professionals, thus decreasing the risk of burnout and second victimhood related to isolation and feelings of inefficacy. Given the association of burnout with decreased empathy
Table 2. Example Workshops

\begin{tabular}{|c|c|c|}
\hline Venues & Speakers & Narrative Themes \\
\hline $\begin{array}{l}\text { Palliative Care } \\
\text { Conference }\end{array}$ & $\begin{array}{l}\text { - Physician } \\
\text { - Social Worker } \\
\text { - Nurse } \\
\text { - Volunteer Services Manager }\end{array}$ & $\begin{array}{l}\text { - Trauma symptoms/PTSD after a } \\
\text { code in the PICU } \\
\text { - Emotional identification with a } \\
\text { young trauma patient who died } \\
\text { - Moral distress related to suffering } \\
\text { at end of life } \\
\text { - Feeling professionally inadequate } \\
\text { due to own serious health issue }\end{array}$ \\
\hline $\begin{array}{l}\text { Child Psychiatry } \\
\text { Grand Rounds }\end{array}$ & $\begin{array}{l}\text { - Two psychologists (one male, } \\
\text { one female) } \\
\text { - Three psychiatrists (two males, } \\
\text { one female) }\end{array}$ & $\begin{array}{l}\text { - Feeling professionally inadequate } \\
\text { due to a patient suicide } \\
\text { - Feeling professionally inadequate } \\
\text { due to the inability to effectively } \\
\text { treat distressing symptoms } \\
\text { - Accidentally sharing a mentee's } \\
\text { unpublished research results } \\
\text { - Being fired from a project and } \\
\text { demoted }\end{array}$ \\
\hline
\end{tabular}

and increased medical errors, quality of care may be positively impacted as well. This paper described a workshop format that can be used to facilitate these goals.

Research is needed to determine if such reflective narrative workshops are effective in attaining the goals outlined above, however preliminary research using videotaped stories by known leaders within neurology residency programs at two institutions is promising [17]. About $80 \%$ of residents found the reflective narrative videos of their respected attendings to be relevant to their personal or professional experience; reported that they learned about coping with physician challenges as a result of viewing the videos; and indicated that they learned about coping with work related challenges as a result of viewing the videos. In the same study, $68 \%$ reported that they gained new perspectives/insights on resiliency from viewing the videos. Most impressive, $89 \%$ of the residents indicated that the videos helped them humanize the attending physicians who shared their videotaped stories. Anecdotal evidence, in the form of verbal feedback following the two example workshops described in the previous section, suggests that live reflective narrative workshops may be even more impactful than the videotape versions with some participants finding the workshop quite emotionally profound, significantly shifting their perceptions of respected leaders in a helpful way.

As emerging research is demonstrating the significant need for health systems to better address health professional burnout and trauma, innovative approaches to impacting local medical setting culture are needed to aid the successful adoption of peer support programs and resilience skills training. Engaging local leaders in this effort is likely to be an essential component of all comprehensive programs. Reflective narrative workshops with known leaders sharing their stories of challenge may be a potent tool to include in larger strategic plans to improve health professional and patient well-being.

\section{References}

1. Moss M, Good VS, Gozal D, Kleinpell R, Sessler CN (2016) A Critical Care Societie collaborative statement: burnout syndrome in critical care health-care professionals. A call for action. American Journal of Respiratory and Critical Care Medicine 194 106-113.

2. Bianchi R, Schonfeld IS, Laurent E (2015) Is it Time to Consider the "Burnout Syndrome" A Distinct Illness? Front Public Health 3: 158. [Crossref]

3. Lee J, Lim N, Yang E, Lee SM (2011) Antecedents and consequences of three dimensions of burnout in psychotherapists: A meta-analysis. Professional Psychology: Research and Practice 42: 252. 

culture

4. Scott SD, Hirschinger LE, Cox KR, McCoig M, Hahn-Cover K, et al. (2010) Caring for our own: deploying a systemwide second victim rapid response team. Jt Comm J Qual Patient Saf 36: 233-240. [Crossref]

5. Fahrenkopf AM, Sectish TC, Barger LK, Sharek PJ, Lewin D, et al. (2008) Rates of medication errors among depressed and burnt out residents: prospective cohort study. BMJ 336: 488-491. [Crossref]

6. Shanafelt TD, Balch CM, Bechamps G, Russell T, Dyrbye L, et al. (2010) Burnout and medical errors among American surgeons. Ann Surg 251: 995-1000. [Crossref]

7. Edward KL (2005) The phenomenon of resilience in crisis care mental health clinicians. Int J Ment Health Nurs 14: 142-148. [Crossref]

8. Halbesleben JR (2006) Sources of social support and burnout: a meta-analytic test of the conservation of resources model. J Appl Psychol 91: 1134-1145. [Crossref]

9. Woodhead EL, Northrop L, Edelstein B (2016) Stress, Social Support, and Burnout Among Long-Term Care Nursing Staff. J Appl Gerontol 35: 84-105. [Crossref]

10. Abaza MM, Nelson KG (2018) Leading by Example: Role Modeling Resilience Helps Our Learners and Ourselves. Acad Med 93: 157-158. [Crossref]
11. Avanzi L, Schuh SC, Fraccaroli F, van Dick R (2015) Why does organizational identification relate to reduced employee burnout? The mediating influence of social support and collective efficacy. Work \& Stress 29: 1-10.

12. Fioretti C, Mazzocco K, Riva S, Oliveri S, Masiero M (2016) Research studies on patients' illness experience using the Narrative Medicine approach: a systematic review. BMJ Open 6: $\mathrm{e} 011220$.

13. Frisina PG, Borod JC, Lepore SJ (2004) A meta-analysis of the effects of written emotional disclosure on the health outcomes of clinical populations. J Nerv Ment Dis 192: 629-634. [Crossref]

14. Winkel AF, Yingling S, Jones AA, Nicholson J (2017) Reflection as a Learning Tool in Graduate Medical Education: A Systematic Review. J Grad Med Educ 9: 430-439. [Crossref]

15. Wimsatt LA, Schwenk TL, Sen A (2015) Predictors of depression stigma in medica students: potential targets for prevention and education. Am J Prev Med 49: 703-714. [Crossref]

16. Hankir AK, Northall A, Zaman R (2014) Stigma and mental health challenges in medical students. BMJ Case Rep 2014. [Crossref]

17. Wald HS, Bursch B (2018) Faculty Videos - An Innovation within Residency Resilience Skills Programs at Two Institutions. In the Accreditation Council for Graduate Medical Education Annual Educational Conference, Orlando, FL, March 2018.

Copyright: $\left({ }^{2} 2018\right.$ Emerson ND. This is an open-access article distributed under the terms of the Creative Commons Attribution License, which permits unrestricted use, distribution, and reproduction in any medium, provided the original author and source are credited. 